29 years (IQR 25-37). HPV vaccination intention was relatively high (mean 2.0; 95\% CI:1.8-2.2). In multivariable analysis attitude $(\beta=0.6 ; 95 \% \mathrm{CI}: 0.5-0.7)$, descriptive norm $(\beta=0.3 ; 95 \%$ CI:0.2-0.4), self-efficacy $(\beta=0.2 ; 95 \%$ CI:0.1$0.3)$, beliefs $(\beta=0.1 ; 95 \% \mathrm{CI}: 0.0-0.2)$ and anticipated regret $(\beta=0.1 ; 95 \%$ CI:0.0-0.2) were the strongest predictors of HPV vaccination intention. Demographic variables did not improve the multivariable regression model. The explained variance in the model (R2) was 0.54 . HPV vaccination intention decreased significantly when vaccination would require out-of-pocket payment (€50 mean: 1.2 (95\%CI: 0.8-1.7); $€ 100$ mean: 1.6 (95\%CI: 1.1-2.0); $€ 200$ mean: $1.0(95 \%$ CI: $0.5-1.5$ ); $€ 350$ mean: 0.2 (95\%CI: $-0.2-0.7$ ).

Conclusion HPV vaccination intention among FSW in Amsterdam appears to be very high. The included socio-psychological factors explained most of the variance in HPV vaccination intention among FSW. Out-of-pocket payment had a significant negative effect on HPV vaccination intention.

\section{P5.21 FISHERMEN AS A SUITABLE POPULATION FOR HIV INTERVENTION TRIALS}

${ }^{1}$ Njeri Mbugua, ${ }^{2}$ Elizabeth Ann Bukusi. 'Kenya Medical Research Institute/Nairobi University/ Kenyatta Hospital/Kenya Women with HIVIAIDS; ${ }^{2}$ Kenya Medical Research Institute, Nairobi University, Kenya

\subsection{6/sextrans-2017-053264.637}

Introduction Suitable populations to sustain continued evaluation of HIV and sexually transmitted infection (STI) prevention interventions are required. We sought to determine whether fishermen are a suitable population for HIV intervention trials.

Methods In a cross-sectional descriptive survey, we selected 250 fishermen from proportional to size sampled boats. We collected socioeconomic and behavioural information, and specimens for HIV, herpes simplex virus (HSV-2), syphilis, gonorrhoea, chlamydia and human papillomavirus (HPV) tests from consenting participants.

Results One third of the fishermen had concurrent sexual partnerships and two thirds were involved in transactional sex. About $70 \%$ were involved in extramarital sex with only one quarter using condoms in their three most recent sexual encounters. HIV prevalence was $26 \%$ and HSV-2 and HPV was $57 \%$. Over $98 \%$ were willing to participate in a future HIV prevention clinical trial.

Conclusion Fishermen are a high-risk group for HIV/STI infections that may be suitable for HIV prevention trials. A cohort study would be useful to measure the incidence of HIV/STIs to ultimately determine the feasibility of enrolling this population in an HIV/STI prevention clinical trial.

\section{P5.22 THE IMPORTANCE OF HAVING AN ADOLESCENT HIV UNIT CASE REPORT AT JOS UNIVERSITY TEACHING HOSPITAL (JUTH), JOS PLATEAU STATE, NIGERIA}

Ogudiegwu Catherine Chinyere. Jos University Teaching Hospital, Nigeria

\subsection{6/sextrans-2017-053264.638}

Issue JUTH has one of the largest HIV treatment centres in North central Nigeria currently has 529 children enrolled into the program,498 on treatment. Since inception in 2004,92 children had virologic failure of their treatment and 45 transferred to the Adult ART program.I present the case of one of our children that have grown into Adulthood who defaulted her treatment,failed treatment regimen, got pregnant from HIV negative sex partner and had her baby is free of HIV. Body: The child was enrolled into the program at age of 10 and she lost mother to HIV when she was 9 years old. Her 1 st line drug regimen was Zidovudine, Niverapine and lamivudine which she was not adherent to.At age 17, she admitted that she had a sexual partner who was later invited counselled and tested, his HIV antibody test was negative.She was later transferred to the adult clinic at the age of 18 years with viral load of 52568 copies/ml while CD4 count of 345 cells $/ \mathrm{mm}$. She got pregnant also immediately from another partner but failed to access Prevention of child transmission of HIV (PMTCT)for fear of being scourned by the health workers. She had a vaginal delivery at 39 weeks.

Intervention Baby was enrolled at the paediatric unit,had Nevirapine prophylaxis for 6 weeks and was breastfeed baby exclusively for 6 months.Condoms use was inconsistent and she was also on daily contraceptive. Her baby recorded HIV negative results for DNA PCR (Deoxyribonucleic acid polymerase chain reaction) results at week 6,12, and 24 and Post breast feeding DNA PCR test.Baby is still incare for followup. Sex patner was counselled,tested for HIV, antibody testing result was HIV negative, and he was subsequently enrolled for the prevention program(PrEP).

Lessons learnt There is need to establish an Adolecent ART clinic where reproductive health can be discussed freely and healthworkers should encourage instead of being judgmental and also we need to educate them on HIV infection and reinfection,drug adherence and also encourage abstinence.

\section{P5.23 HOW MUCH CAN HIV TRANSMISSION BE REDUCED IN HIGH-RISK MSM BY TARGETING TESTING TO DETECT AND TREAT PRIMARY HIV INFECTION (PHI)? ANALYSIS OF A COHORT STUDY USING AN INDIVIDUAL-BASED MODEL}

${ }^{1}$ Peter White, ${ }^{2} J u l i e$ Fox, ${ }^{1} J o n a t h a n$ Weber, ${ }^{1}$ Helen Ward, ${ }^{1}$ Sarah Fidler. ${ }^{1}$ Imperial College London, UK; ${ }^{2}$ Guys and St Thomas' NHS Trust/Kings College London, UK

\subsection{6/sextrans-2017-053264.639}

Introduction HIV incidence remains high in UK MSM, and cost-effective combination prevention is needed. We estimated numbers of infections averted by targeting testing for highlyinfectious primary HIV infection (PHI) in high-risk MSM.

Methods A cohort study of 98 MSM (participation rate 94\%; zero loss to follow-up) recently infected with HIV recorded sexual behaviour in the 3 months pre- and post-HIV diagnosis. A stochastic individual-based model calculated numbers of HIV-transmission events expected to occur with and without the effects of (i) immediate ART in those who chose to receive it and (ii) behaviour changes reported post-diagnosis by those who did and did not receive ART. The model incorporates different types of sex-act, condom use, and distinguishes between regular and casual partners.

Results If PHI lasts for 3 months, testing is monthly, and viral load is suppressed by ART after 3 months with an initial rapid decline in the first 2 weeks, then from the 73 patients who took ART the reduction in transmission would be $\sim 75 \%$, from $22-33$ to $5-8$ events, and in the remaining 25 patients 
the reduction (due to behaviour change only) would be $\sim 50 \%$, from $8-12$ to $5-7$. If the duration of $\mathrm{PHI}$ is shorter, or testing frequency is lower frequency, and/or viralload suppression is slower then the number of infections averted is reduced.

Conclusion Diagnosing HIV during PHI can markedly reduce transmission because its high infectivity and short duration make treatment and even short-term behaviour change effective in reducing transmission. Cost-effectiveness would be increased by efficient approaches to identifying PHI (e.g. encouraging HIV testing after episodes of high-risk behaviour and intensive contact tracing from recently-infected individuals, since PHI cases are likely to be clustered). Our quantification of the number of infections averted is an essential component of assessment of the cost-effectiveness of strategies to increase diagnoses of PHI.

\section{P5.24 DRUG RESISTANCE AMONG WOMEN ATTENDING ANTENATAL CLINIC IN GHANA}

Philip Enyan. University of Ghana, Ghana

10.1136/sextrans-2017-053264.640

Introduction Initial evidence from resource-limited countries using the WHO HIV drugresistance (HIVDR) threshold survey suggests that transmission of drug-resistance strains islikely to be limited. However, as access to ART is expanded, increased emergence of HIVDR isfeared as a potential consequence. We have performed a surveillance survey of transmittedHIVDR among recently infected persons in the geographic setting of Accra, Ghana.

Methods As part of a cross-sectional survey, 2 large voluntary counselling and testing centres inAccra enrolled 50 newly HIV-diagnosed, antiretroviral drug-naïve adults aged 18 to 25 years.Virus from plasma samples with $>1,000$ HIV RNA copies/mL (Roche Amplicor v1.5) weresequenced in the pol gene. Transmitted drug resistance-associated mutations (TDRM) wereidentified according to the WHO 2009 Surveillance DRM list, using Stanford CPR tool (v 5.0beta). Phylogenetic relationships of the newly characterised viruses were estimated bycomparison with HIV-1 reference sequences from the Los Alamos database, by using theClustalW alignment program implemented.

Results Subtypes were predominantly D (39/70, 55.7\%), A $(29 / 70,41.4 \%)$, and C $(2 / 70 ; 2,9 \%)$. Seven nucleotide sequences harboured a major TDRM (3 NNRTI, 3 NRTI, and 1 PIassociated mutation); HIVDR point prevalence was $10.0 \%$ (95\%CI $4.1 \%$ to $19.5 \%)$. The identified TDRM were D67G (1.3\%), L210W (2.6\%); G190A (1.3\%); G190S (1.3\%); $\mathrm{K} 101 \mathrm{E}(1.3 \%)$, and N88D (1.3\%) for PI.

Conclusion In Accra the capital city of Ghana, we found a rate of transmitted HIVDR, which,according to the WHO threshold survey method, falls into the moderate $(5 \%$ to $15 \%)$ category.This is a considerable increase compared to the rate of among women attending an antenatal clinic in mamobi. As ART programs expand throughoutAfrica, incident infections should be monitored for the presence of transmitted drug resistance inorder to guide ART regimen policie.

\section{P5.25 FEASIBILITY OF A POINT-OF-CARE MODEL FOR THE PREVENTION OF MOTHER TO CHILD TRANSMISSION OF SYPHILIS IN CALI, COLOMBIA}

${ }^{1}$ Potes LI, ${ }^{1}$ Rubiano LC, ${ }^{1}$ Ramírez LG, ${ }^{2}$ Tunubalá GA, ${ }^{1}$ Orobio Y, ${ }^{3}$ Salazar JC. ${ }^{1}$ CIDEIM, Colombia; ${ }^{2}$ ESE Norte, Colombia; ${ }^{3}$ University of Connecticut School of Medicine and Connecticut Children's Medical Centre

\subsection{6/sextrans-2017-053264.641}

Introduction Most women in Colombia receive antenatal care (ANC), but goals for the reduction of congenital syphilis (CS) have not been met. In 2015 Cali had an incidence of 3.0 cases of CS per 1000 births.Point of care testing (POC) has proven cost-effective, and since 2015 it is now recommended by the Colombian Ministry of Health Guidelines. Nevertheless, POC for gestational syphilis (GS) has not been implemented in ANC. We sought to test the feasibility of POC and identify barriers to its implementation in Floralia, a public, primary healthcare centre in Cali.

Methods During a three-month period, we observed routine ANC in Floralia and offered women on-site rapid tests (RT) for syphilis. Process measures were compared with data from the clinical charts (CC) of women seen in 2015. Patients and administrators were interviewed and asked to describe ANC, syphilis screening procedures, and to identify opportunities for improvement.

Results 55 women were offered POC testing, 42 agreed to participate and were screened. 55 CC from 2016, and 123 CC from 2015, were reviewed. The median gestational age at which women sought health care was eight weeks. The first trimester of pregnancy had the highest percentage of women screened for GS: $97 \%$ were screened in 2015, and $100 \%$ were screened in 2016. Changes to screening procedures were hindered by existing contracts with insurance companies (IC); they determine what tests can be ordered and who can order them. Administrative procedures lead to missed opportunities for screening and treatment.Two women (1.6\%) from the 2015 group were diagnosed with GS, and three (5.4\%) were diagnosed during the intervention. POC testing allowed us to treat GS on the same day of the positive result, while using routine testing methods took 6 to 24 days.

Conclusion Diffusion of the national guidelines for GS has been insufficient to control GS and CS: There is still confusion among health care workers, and contracts with IC do not include RT. POC for syphilis can be a part of ANC, but barriers need to be addressed before implementation.

\section{P5.26 ATYPICAL PAP SMEAR BEFORE AND AFTER HPV VACCINE IN PRIVATE SERVICE AT FORTALEZA, BRAZIL}

${ }^{1}$ Renata Mirian Nunes Eleuterio, ${ }^{1} J o s e ́$ Eleutério Junior, ${ }^{1}$ Ana Larissa Duarte, ${ }^{2}$ Paulo Giraldo,

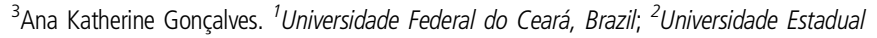
de Campinas - UNICAMP, Brazil; ${ }^{3}$ Universidade Federal do Rio Grande do Norte, Brazil

\subsection{6/sextrans-2017-053264.642}

Introduction HPV vaccine is a powerful tool in to prevent cervical cancer. It were commercially introduced in Brazil in 2008 and only in 2013 the government starts in public health.Objectives: To assess the influence of the introduction of HPV vaccine in Fortaleza (Brazil) in the results of Pap smear.

Methods The results of Pap smear between 2006 and 2014 in Prof. Eleutério Laboratory were searched in the files to 